\title{
ESPACIOS, EXPERIENCIA DE OCIO Y PARTICIPACIÓN DE LA JUVENTUD: CONTRIBUCIÓN A LOS MODELOS DE GESTIÓN E INTERVENCIÓN A PARTIR DEL ANÁLISIS DE BUENAS PRÁCTICAS ${ }^{1}$
}

\author{
Joseba Doistua Nebreda \\ Universidad de Deusto \\ Héctor Manuel Pose Porto \\ Universidad de La Coruña \\ Ruth Ahedo González \\ Universidad de Deusto
}

\begin{abstract}
RESUMEN: Se entiende la juventud como un proceso de experimentación en el cual se generan las bases sobre las cuales se sustentará, en buena medida, la vida adulta. En este sentido, el ocio se presenta como un escenario idóneo para la experimentación, principalmente por la libertad en la elección del mismo. En este texto se realiza un análisis de experiencias y proyectos que pueden ser consideradas como buenas prácticas en el contexto comunitario, tanto desde el punto de vista de las actividades y prácticas de ocio que se realizan, como desde el punto de vista de los diferentes modelos de gestión e intervención. En el análisis se investigan principalmente los diferentes modelos de participación de la juventud en el diseño y gobernanza del ocio y cómo este aspecto contribuye al desarrollo personal y social del propio joven. Además, también se analiza cómo este aspecto puede favorecer el desarrollo de una serie de actitudes y competencias para la participación social, política y cultural en su barrio, municipio o comarca. En general, se puede concluir que a una mayor implicación del joven en el diseño y gestión de la oferta comunitaria de ocio, mejor es la experiencia de dicha actividad y el grado de compromiso y participación en el programa o servicio.

PALABRAS CLAVE: Buenas prácticas, juventud, participación juvenil, ocio, espacio, modelo de gestión.
\end{abstract}

1. El texto que presentamos se vincula al subproyecto "De los tiempos educativos a los tiempos sociales: Participación de los jóvenes en la creación y gobernanza de sus espacios de ocio como factor de desarrollo personal" (EDU2012-39080-C07-03) incluido dentro del Proyecto de Investigación "De los tiempos educativos a los tiempos sociales: La construcción cotidiana de la condición juvenil en una sociedad de redes. Problemáticas específicas y alternativas pedagógico-sociales" (proyecto coordinado EDU201239080-C07-00), cofinanciado en el marco del Plan Nacional I+D+i con cargo a una ayuda del Ministerio de Economía y Competitividad, y por el Fondo Europeo de Desarrollo Regional (FEDER, 2007-2013). 


\title{
SPACES, LEISURE EXPERIENCE AND YOUTH PARTICIPATION: A CONTRIBUTION TO THE MANAGEMENT AND INTERVENTION MODEL BASED ON THE ANALYSIS OF THE BEST PRACTICES
}

\begin{abstract}
Youth is understood as an experimentation process during which the main bases that will underpin adult life are created. Accordingly, leisure can be regarded an ideal scenario for experimentation, mostly due to the freedom of choice it involves. This text contains an analysis of experiences and projects that may be considered good practices in the community context, from the perspective of the leisure activities and practices carried out as well as from the point of view of the various management and intervention models. The analysis mainly centers on the different participation models of young people themselves in leisure design and governance and how this aspect contributes to their personal and social development. Furthermore, it analyses how this can foster development of a series of attitudes and competences for social, political and cultural participation in their neighborhoods, cities or districts. In general terms, the conclusion is that the more young people are involved in the design and management of community leisure activities, the better their experience of such activities, degree of engagement and participation in the program or service are.

KEYWORDS: Good practice, youth, youth participation, leisure, management model.
\end{abstract}

Recibido: 09/05/2015

Aceptado: 22/07/2015

Correspondencia: Joseba Doistua Nebreda, Instituto de Ocio de la Universidad de Deusto, Facultad de Ciencias Sociales y Humanas, Campus Bilbao, Avenida de las Universidades 24, 48007 Bilbao. Email: joseba.doistua@deusto.es.

\begin{abstract}
AnTECEDentes
Hoy en día es necesario comprender y valorar en su justa medida la importancia que tiene la juventud como proceso de construcción personal y social. En la investigación actual, la centralidad del colectivo de personas jóvenes es cada vez mayor y en este sentido, para López (2010: 235) "Ios jóvenes reflejan los cambios a los que la sociedad va dando lugar". Asimismo, el filósofo Aristóteles planteaba que "Adquirir desde jóvenes tales o cuales hábitos no tiene poca importancia: tiene una importancia absoluta" (Aristóteles, EN II, 1, 1003 b 25. Trad. 1970). La centralidad del joven en la investigación social actual debe trabajarse desde criterios científicos y no tener en cuenta a los agentes que intervienen en juventud o del propio colectivo joven sería un error. Es por ello que el punto de partida de este texto busca conocer, a través de experiencias que han sido consideradas como buenas prácticas por un equipo multidisciplinar, qué repercusiones tiene en la experiencia de ocio de la juventud la gestión e intervención en los espacios juveniles y su desarrollo personal y social a través de estas vivencias.
\end{abstract}


La juventud es entendida como un espacio social de experimentación individual prolongado, donde conviven una multiplicidad de situaciones en las que se vuelve difícil encontrar términos que den cuenta de esa complejidad que ya no es exclusiva de lo que ha venido entendiéndose por juventud (Gil Calvo, 2005; Tejerina, Carbajo y Martínez, 2012).

Se comparte en este artículo buena parte de los objetivos que se plantean Ortega, Lazcano y Manuel (2015), al buscar en las políticas de juventud el papel que juega la autogestión y gobernanza de los espacios juveniles y de ocio desde la propia juventud y el papel que tienen los agentes que intervienen en juventud.

Se entiende que la participación de las personas jóvenes en los procesos de creación y gobernanza de los espacios formales e informales de ocio es una dimensión imprescindible en el estudio de la construcción cotidiana de la condición juvenil, sobre todo, ya que para este colectivo la importancia otorgada al ocio ha ido aumentando en los últimos años tal y como señala López (2010).

Es en la juventud donde se marcan de forma más profunda las bases sobre las cuales se sustentará la vida adulta. Se trata de un periodo en el cual la persona se enfrenta a enormes retos: oportunidades y peligros de la vida real. En la actualidad, el consumo entre los jóvenes, no solo en su vínculo con el ocio, sino por constituirse en un elemento de estatus ante sus grupos de referencia, provoca un desarrollo tendente a provocar desequilibrios en la formación del joven. Asimismo, Caride (2012: 309) señala que "las experiencias de ocio, en su inscripción en los nuevos tiempos sociales y los riesgos que comportan en una sociedad espacio-temporal transformada, están siendo objeto de un profundo debate intelectual y social".

Además, hoy en día, la importancia del fenómeno del ocio resulta innegable en la definición de la sociedad y las personas. Pero esta experiencia puede vivenciarse de formas muy diferentes e influye determinantemente en la vida de la juventud. Es por ello que el análisis de los diferentes modelos de intervención en los espacios juveniles cobra cada vez una mayor importancia.

El grupo de pares, las amistades, son una gran referencia para las personas jóvenes tal y como se puede constatar a través de diferentes estudios. Como señalan Doistua, Cuenca y Ortega (2014), a partir de los datos de la investigación sobre las prácticas y experiencias de ocio de la juventud en la Comunidad Autónoma de Euskadi, de las particularidades que se pueden encontrar en el ocio de la juventud, el papel de su grupo de pares es determinante. En dicho estudio se constata que las actividades de ocio que realizan con sus amistades llegan al 65,3\% del total de su práctica de ocio. Por otro lado, el conjunto de actividades de ocio que la juventud realiza a través de asociaciones o clubes supone sólo el 5,3\% del total de prácticas realizadas. Estos datos constatan el hecho de que para el joven el grupo de amistades determina en gran medida su práctica de ocio, así como la experiencia que pueda desarrollar a partir de dichas actividades.

Como señalan Rodríguez, Megías y Sánchez (2002) con los amigos se comparte lo malo (lo relacionado con los problemas íntimos) y lo bueno (la diversión de la marcha), pero el grupo de marcha no está más implicado necesariamente por relaciones de amistad, es más, las relaciones que constituyen la intimidad de amigos cuando coinciden en los tiempos y espacios de diversión, se diluyen en el grupo. 
Por lo tanto, por un lado se puede observar cómo las personas jóvenes en su ámbito de ocio otorgan una importancia grande a sus amistades. Por otro lado, vemos cómo uno de los principales problemas con el que se encuentran las políticas de intervención en juventud es el acceso a este colectivo, ya que los espacios que ocupan para el desarrollo de buena parte de su ocio es un espacio que tiene un carácter privado o, al menos, separado de otros colectivos. Hoy en día coexisten políticas de juventud que atienden directamente a la juventud en el espacio público abierto (calle, plazas, etc.) o en sus espacios de carácter privado, con políticas de juventud que generan espacios propios para el colectivo joven.

Las políticas de juventud en materia de ocio atienden a toda la tipología de espacios dirigidos a este colectivo. En los últimos años se han elaborado distintas clasificaciones y en ese sentido este texto toma como guía la clasificación de los profesores Berrio-Otxoa, Hernández y Martínez (2002). Ésta identifica cuatro espacios: compartidos, propios, monitorizados y reciclados. Espacios limitados por un eje de dos polos: heteronomía y autonomía. Las relaciones, los espacios y las actividades que el colectivo de personas jóvenes sitúa en el extremo de autonomía son las que viven con más placer en su tiempo libre, mientras que en el extremo de la heteronomía o dependencia son las que la mayoría desea evitar. Los espacios heterónomos se definen como espacios ordenados y disciplinados, dirigidos por adultos cuyo funcionamiento está controlado también por adultos. Por el contrario, los espacios autónomos serían aquellos espacios desregularizados y flexibles, apartados de la mirada de los adultos, normativizados y gestionados por adolescentes.

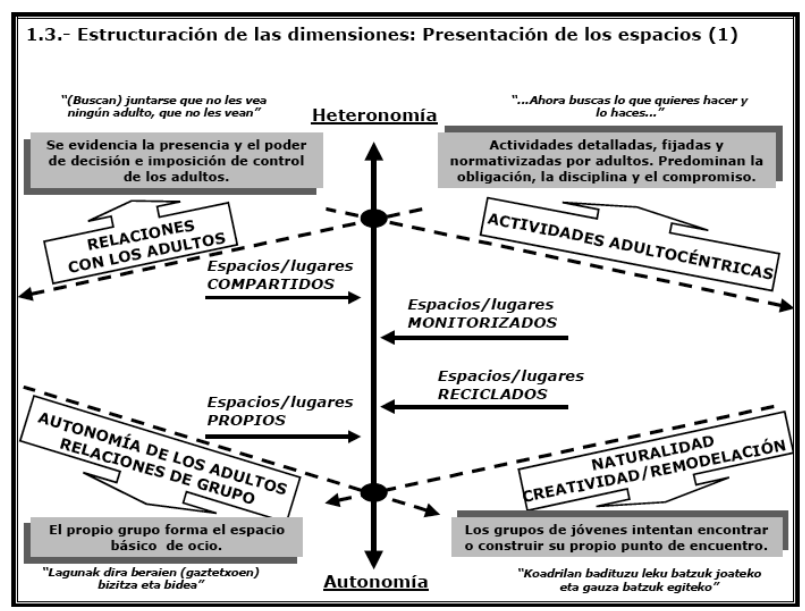

Figura 1. Modelo de clasificación de los espacios donde la juventud desarrolla sus actividades en función del grado de autonomía frente a las personas adultas.

Fuente: Berrio-Otxoa, Hernández y Martínez (2002)

Como señala Tejerina et al. (2012), más allá de las críticas posibles a esta tipología, lo que resulta importante destacar es su capacidad para hacer comprensible los espacios de ocio joven dentro de un continuo que va desde una normativización adulta e institu- 
cional (heterónoma para la juventud) a una normativización (emergente, institucionalizante, autónoma) elaborada desde los propios jóvenes. Es decir, posibilita, de manera transversal, identificar un proceso de diferenciación (y constitución) de los espacios juveniles y sus conexiones con la construcción de la identidad grupal (y personal).

La participación de los jóvenes en los procesos de creación y gobernanza de los espacios informales de ocio es una dimensión imprescindible en el estudio de la construcción cotidiana de la condición juvenil. Esto da lugar a fenómenos de alcance político, social y educativo tales como lonjas, locales, sociedades o peñas, según el contexto geográfico. Pero también en relación a los espacios formales o institucionalizados de práctica de ocio juvenil como pueden ser los espacios jóvenes o centros cívicos o culturales donde se reservan espacios para la práctica de ocio de la juventud.

Para Tejerina et al. (2012) el "estar juntos" es la clave. El grupo de pares establece dos dimensiones fundamentales, por un lado, presenta una dimensión interna como grupo humano que genera, en su privacidad, una complicidad grupal (es decir, una tendencia gregaria que cohesiona al grupo), la interpretación de experiencias, formación de gustos comunes y referencias, entre otras cosas, para el uso del tiempo de ocio. Por otro lado, la cuadrilla tiene una dimensión externa o pública y se constituye como una institución social que permite a los individuos vivir colectivamente e integrarse en la vida social. En este ambiente, los jóvenes construyen una identidad personal y social, ya que las lonjas ${ }^{2}$ refuerzan los vínculos de amistad y permiten desarrollar la autonomía personal y privacidad.

Habiendo identificado experiencias y proyectos que podemos considerar como buenas prácticas en el contexto comunitario, en este texto se pretende analizar cómo estas se relacionan con los espacios de ocio, tanto desde el punto de vista de las actividades y prácticas que se realizan, como desde el punto de vista de los diferentes modelos de gestión e intervención.

Dentro del subproyecto "De los tiempos educativos a los tiempos sociales; la participación de los jóvenes en la creación y gobernanza de sus espacios de ocio como factor de desarrollo personal", que presenta el Grupo de Investigación constituido en la Universidad de Deusto (UD), se investiga el modo en que se generan y gestionan estos espacios y los tiempos que habilitan e influyen de forma decisiva en el mayor o menor alcance socioeducativo de tales prácticas, poniendo de relieve el interés y la importancia que supone no solo conocerlas sino también transformarlas con propósitos educativos y culturales.

Este texto pretende dar respuesta en cierta medida a dicho objetivo centrándose en la lectura de cómo las buenas prácticas gestionan los espacios físicos donde la juventud desarrolla su ocio. Bien porque se tratan de espacios de titularidad pública, espacios al aire libre o espacios de la propia juventud. Otra de las preguntas a las que se pretende responder es cómo se plantean desde estos proyectos de intervención los objetivos de ocio en relación a los usuarios. Finalmente, se hará hincapié en el papel que el joven juega en la creación, definición o gestión de dichos espacios y qué beneficios y problemáticas tiene cada tipo de gestión en relación a los espacios.

2. Este término hace referencia en la Comunidad Autónoma de Euskadi a lo que serían los locales juveniles autogestionados y de carácter privado tales como sociedades, txokos, locales de cuadrilla, bajeras, etc., según el contexto geográfico del que se hable. 


\section{Diseño DE LA INVESTIGACIÓN}

Como se ha señalado anteriormente, este texto surge del proyecto RESORTES, que tiene como objetivo general "estudiar la naturaleza y el alcance de los tiempos educativos y sociales en la construcción cotidiana de la condición juvenil, analizando de qué forma y en qué grado inciden en la vida de jóvenes pertenecientes a distintos contextos y realidades, identificando sus problemáticas específicas y las alternativas que deberán adoptarse para contribuir al desarrollo integral de su personalidad y al más pleno ejercicio de sus derechos cívicos". Partiendo de este objetivo general se estableció como una de las líneas de trabajo la identificación de experiencias significativas relacionadas con los tiempos educativos y sociales de la juventud, en clave pedagógica, psicológica y sociológica, que orienten un marco estratégico de reflexión-acción en este campo.

\section{Diseño metodológico}

El análisis de buenas prácticas se ha llevado a cabo a través de un diseño metodológico que se enmarca dentro del paradigma interpretativo (González-Monteagudo, 2000), siguiendo una metodología cualitativa. Se trata de una metodología compleja en la que una de las primeras técnicas planteadas es el análisis de experiencias desarrolladas en este ámbito, realizadas por diferentes agentes que intervienen en el campo del ocio y de los tiempos sociales de la juventud. Tal y como expresan Ahedo, Valdemoros, Escolar, Melendro, Serrat y Pose (2014), es muy importante para el proyecto RESORTES visibilizar y poner en valor las buenas prácticas que toman como soporte programas y proyectos promovidos por los entes promotores observadores, administraciones públicas, instituciones, asociaciones, etc.

Una de las riquezas de la búsqueda de buenas prácticas por parte de los siete equipos que conforman este grupo de investigación es la autonomía que se confirió a los diferentes equipos en la búsqueda de dichas buenas prácticas. Tal y como se señala en dicho texto, citando a Caride, Fraguela y Valera (2014), el nexo de unión de dichos equipos es la lealtad a los principios del ocio humanista y la creencia de la contribución del ocio al desarrollo humano (Cuenca, 2000), si bien cada equipo de investigación tiene diferentes ámbitos de investigación que otorgan una complementariedad a todos los análisis y trabajos realizados. Eso se logra al poder analizar cada una de las buenas prácticas desde un punto de vista concreto que, en este caso, es el papel que el espacio y su gobernanza tienen en la experiencia de ocio y en la participación juvenil en dichos programas y espacios.

\section{Procedimiento}

El proceso de selección de las buenas prácticas se llevó a cabo a través de las siguientes fases (Ahedo et al., 2014):

En la primera fase se trabajaron los criterios e indicadores que ayudan a clarificar y distinguir una buena práctica. Entre estos criterios destacaban: el carácter innovador del servicio prestado, la estructura organizativa, la transferibilidad, la viabilidad del proyecto, su transversalidad, el impacto positivo en su ámbito de 
actuación, su permanencia en el tiempo, la sostenibilidad, la eficacia y eficiencia del proyecto o servicio, o un completo sistema de evaluación.

Una vez compartidos y decididos dichos criterios, se pasa a una segunda fase en la que cada grupo de investigación se encargó de identificar experiencias de buenas prácticas, hacer una recogida de los datos en base a una ficha base común para todos los equipos y posteriormente enviarlo al resto de equipos. En esta fase se recopilaron 21 experiencias de buenas prácticas que fueron sometidas a un proceso de revisión por parte de todos los equipos, seleccionando aquellas que cumplían los criterios preestablecidos. Asimismo, se comprobó que no quedara ningún ámbito temático o territorial que fuera de interés y que no se hubiera abordado.

La tercera fase permitió la depuración de los datos recogidos, se unificaron los criterios de redacción y aspectos de carácter más formal. La cuarta fase supuso la selección final de las 19 experiencias que componen la base de datos final de buenas prácticas del proyecto RESORTES. Finalmente, la quinta fase hace referencia a la divulgación de los datos recogidos, a través, por un lado de su inventario en la página web del proyecto, previa autorización y revisión de los agentes que desarroIlan dichas prácticas. Asimismo, se planteó la necesidad de realizar una difusión de dichos contenidos a través de la participación en congresos y jornadas científicas, así como a través de artículos que analizaran parcialmente las experiencias a través de los diferentes prismas planteados por los equipos de investigación de la red. Este texto es un ejemplo de ello.

\section{Muestra}

Teniendo en cuenta las necesidades del proyecto RESORTES, la selección de las buenas prácticas debía abarcar diferentes ámbitos de intervención. Sin embargo, para la realización de este texto se han tenido en cuenta aquellas que guardan relación con la temática de estudio planteada en los antecedentes, y que hacen referencia a los espacios y la experiencia de ocio a través de diferentes modelos de intervención y gestión. Por tanto el análisis que se va a realizar a continuación se ha centrado únicamente en 7 de las buenas prácticas identificadas. Estas experiencias son Quart Jove, Esta es una plaza, Programas de Centros Jóvenes Municipales, Estudio sobre la atención a los jóvenes extutelados en Cataluña, Comarkalada, Giltzarri. Servicio municipal de ocio educativo juvenil y Jóvenes y lonjas. Lugares comunes. Territorialmente son experiencias de Valencia, Madrid, Logroño, Cataluña, comarca de Noguera, Barakaldo y Portugalete.

\section{ANÁLISIS DE LAS BUENAS PRÁCTICAS Y SU DISCUSIÓN}

Para el análisis de las buenas prácticas se toma como hipótesis de partida algunas de las reflexiones que Ortega et al. (2015) plantean en sus conclusiones. Por un lado, se señala que las personas jóvenes encuentran más beneficios en lugares donde adquieren una mayor autonomía y oportunidad de autogestión, ya que se sienten más libres al sentirse lejos de la mirada de los adultos, incluyendo los espacios monitorizados que pese a tener una figura adulta, ésta es cercana y se presenta como persona de referencia e incluso confidente. 
Otra conclusión importante para este análisis es que los entornos influyen en la vivencia de los espacios, ya que un entorno rural es, a priori, donde se dan las relaciones más cercanas y estrechas, mientras que el entorno urbano es más flexible, superficial y cambiante. Esto influye a la hora de diseñar los espacios juveniles y gestionar la oferta vinculada a dichos espacios.

La tercera conclusión a la que llegan Ortega et al. (2015) hace referencia a las actividades que se promueven desde los espacios juveniles. Se observa que tienen más éxito entre las personas jóvenes aquellas que no requieren de un compromiso permanente y aquellas que han sido organizadas y creadas previamente por los jóvenes.

Además de este tipo de recomendaciones, Ortega et al. (2015) señalan una serie de aspectos clave a abordar en los espacios dirigidos al colectivo joven como son la atención a todas las etapas del ciclo vital del joven, promover un lugar donde los jóvenes puedan desarrollar su actividad, fomentar la participación y cooperación, incentivar que los y las jóvenes sean los protagonistas del proceso y participen o coparticipen activamente en la dirección, diseño, programación, gestión, ejecución y evaluación de las actividades, además de tener en cuenta el entorno y fomentar la relación entre pares.

Teniendo en cuenta estas premisas, de las 19 experiencias consideradas como buenas prácticas, 7 de ellas plantean los aspectos recogidos en los antecedentes de este texto, por lo tanto pueden ser utilizadas para comprobar hasta qué punto las hipótesis que se plantean al inicio de este apartado se pueden contrastar con la realidad de los servicios y programas que están enfocados a la población joven y su ocio.

Atendiendo a los promotores de las buenas prácticas analizadas, encontramos que en cinco de ellas los promotores son los ayuntamientos, principalmente a través de sus áreas de juventud. De las dos excepciones una se trata de un consorcio en el que además de agentes públicos también participan asociaciones juveniles y en otro caso se trata de una asociación cultural del barrio madrileño de Lavapiés.

Uno de los aspectos que se detallan en las fichas y que aporta información de interés es el apartado que hace referencia a las palabras clave. La palabra clave más utilizada, después de juventud, es participación. Buena parte de los proyectos no entiende la experiencia sin la necesaria participación del joven a todos los niveles, no sólo como receptores del servicio o programa, sino como parte activa de su diseño, ejecución, gestión y evaluación. De las diferentes experiencias se pueden extraer frases tales como "entre los objetivos del programa se encuentra el promover la participación e implicación de los jóvenes en el proceso organizativo de las actividades" (Comarkalada) o "impulsar y apoyar la autonomía personal y grupal de los chicos y chicas desarrollando sus competencias para el diseño y desarrollo de iniciativas sociales, socio-culturales y de solidaridad" (Giltzarri). Entienden, por tanto, que el éxito del programa depende de la involucración del joven en todo el proceso. Como último ejemplo, se puede señalar que el programa Jóvenes y Lonjas indican que "la participación en la gestión de lo que se percibe como propio genera responsabilidad. Uno gestiona de una determinada manera lo suyo o lo que le interesa para no perderlo... Conociendo los límites será más fácil que asuman su responsabilidad".

Además de la necesaria corresponsabilidad, la integración de todos los espacios y programas orientados a la juventud cobra una importancia vital en el diseño de los 
programas y servicios, siendo conscientes de que la intervención en el ámbito joven debe ser lo más transversal posible. En ese sentido, desde Quart Jove se señala que "la relevancia de la propuesta es precisamente su carácter integral, es decir, el modo en que los distintos espacios de uso juvenil y los distintos programas que se desarrollan se engarzan entre sí con el factor común de la autogestión, la discusión y la defensa de las distintas propuestas en los ámbitos de decisión comunitaria establecidos a tal efecto". En el proyecto Esta es una plaza, destacan que la experiencia "ha adquirido una importante y creciente relevancia desde sus inicios, configurándose como un organismo vivo en el que se exploran y ejercitan la práctica social y los procesos participativos ciudadanos en múltiples direcciones y sentidos".

Entre las hipótesis de partida, se planteaba como un factor positivo el hecho de que un buen planteamiento de las actividades de ocio daba como resultado una mayor continuidad de los usuarios de dichas experiencias. Es decir, este buen planteamiento incluye facilitar la participación, corresponsabilidad y la inclusión de todos los espacios y tiempos que afectan a la persona joven. Teniendo en cuenta las buenas prácticas analizadas, se observa que el programa más joven es de 2008, remontándose algunos a 1994 o 1995. Lo que constata no sólo la continuidad a lo largo del tiempo, sino también el alto grado de participación de jóvenes en dichas experiencias. Dentro del campo de las fichas de buenas prácticas en el apartado que recoge los aspectos a destacar por los organizadores, la mayoría señala "la alta participación juvenil", "la participación", "oferta estable y permanente en el tiempo", "transversalidad", "fomento de actividad", "la sistematización de la gestión integral de los centros como recurso para los barrios y la ciudad", etc.

La importancia que para cualquier proyecto o programa tiene el éxito de participación es clave. Permite su continuidad, mejora progresiva y eficiencia, ya que pese a una posible reducción presupuestaria, el compromiso de los diferentes agentes, así como de los participantes, mantiene la calidad y continuidad del proyecto. Así lo señalan en las experiencias recogidas: "Aunque actualmente el presupuesto económico destinado a la actividad se ve afectado por el contexto, no se cuestiona la continuidad de la práctica" (Giltzarri); "todas las actividades desarrolladas ha tenido un carácter completamente gratuito y en momentos de dificultad se ha estimulado la creatividad y las relaciones" (Esto es una plaza), "año tras año ha crecido el número de socios, la asistencia diaria y el número de actividades ofrecidas" (Centros Jóvenes Municipales), "durante más de 20 años se ha ofrecido el servicio y nadie duda de su continuidad" (Quart Jove).

Un aspecto interesante es comprobar cómo este tipo de experiencias desarrollan y potencian los vínculos entre todas las partes. Por un lado, refuerzan las relaciones entre los propios jóvenes, por otro lado entre las personas jóvenes y la comunidad y finalmente entre la juventud y las instituciones públicas como ayuntamientos, así como entre diferentes agentes que intervienen en juventud. El proyecto de Jóvenes y Lonjas destaca como resultados el "acercamiento entre jóvenes y Alcalde", "establecimiento de un vínculo que facilita posteriores intervenciones en otros proyectos", "puesta en marcha de habilidades personales e interpersonales" y la "mejora de la convivencia ciudadana". Desde el proyecto Esto es una plaza, se destaca que "se han desarrollado más de 100 actividades que han contribuido a fortalecer el tejido social del barrio y a integrar a colectivos desfavorecidos, estimulando la creatividad 
de vecinos y vecinas". Desde el proyecto Giltzarri, plantean que "el Ayuntamiento, desde una perspectiva territorial de la ciudad, desarrolla los procesos participativos y socio educativos en el tiempo de ocio juvenil, desde un estilo de intervención muy definido". Por último, es interesante ver que Quart Jove destaca "la implicación de las instituciones de educación formal y la utilización de estas de los recursos municipales para la juventud, así como la alta coordinación y colaboración con el resto de departamentos municipales".

Uno de los principales objetivos de este texto es destacar la importancia que tienen los espacios en estos resultados. En el caso de Giltzarri se señala la importancia de "estar presentes en los espacios juveniles, en los barrios, en los centros educativos, allí donde están los y las adolescentes, en su territorio, para partir de su propio mundo, de sus motivaciones y necesidades". El programa de Centros Jóvenes Municipales indica la importancia de mantener las actuales infraestructuras y crear nuevos centros que cubran todas las necesidades". Esto es una plaza presenta su espacio como "un organismo vivo en el que, de acuerdo con los ritmos propios de trabajar en un espacio al aire libre, han puesto en marcha un proceso muy parecido a lo que pasa en la naturaleza". Finalmente, la experiencia de Jóvenes y Lonjas destaca que "las personas, tradicionalmente han buscado espacios de uso recreativo privado en grupo, al margen de su propia vivienda. Espacios donde estar, donde celebrar, donde encontrarse e intercambiar o compartir intereses, gustos e inquietudes... Esta búsqueda de privacidad compartida con iguales no es más extraña entre los/las jóvenes que entre los adultos. La diferencia puede estar en que para ellos/as supone contar con un espacio alejado del control de sus adultos".

Todos estos proyectos señalan en las fichas de recogida de datos, los impactos positivos que estas acciones tienen sobre el joven y la sociedad en general, entre las que podemos destacar de forma global las siguientes, más o menos compartidas por todas las experiencias:

- Alto porcentaje de participación de los jóvenes en la vida municipal a través de estos proyectos.

- Aumento de la vida cultural, deportiva y de ocio de la juventud.

- Mejora en la preparación y en el desarrollo de competencias por parte de las personas jóvenes. Empoderamiento juvenil.

- La imagen de la labor de las diferentes áreas municipales implicadas, así como de los técnicos y mediadores mejora considerablemente.

- Modelos de intervención basados en la convivencia, negociación, autogestión, etc.

- El éxito de dichas experiencias garantiza su continuidad.

- Crece el grado de asociacionismo cultural de la juventud.

Finalmente, en este apartado de análisis de las experiencias de buenas prácticas es necesario mostrar las perspectivas de futuro que se plantean y que forma parte de las fichas de recogida de datos. A modo de ejemplo, el proyecto Jóvenes y Lonjas, señala que "en la medida en que se va consolidando el programa en el municipio, se pretende que las personas usuarias del mismo participen como ciudadanía ac- 
tiva y propositiva". La experiencia Esta es una plaza de cara al futuro se plantean "seguir fortaleciendo este espacio de convivencia que ahora habita, porque piensan que es un espacio que contribuye de una forma evidente en la mejora del entorno urbano, tanto a nivel medioambiental, como a nivel social (creando tejido asociativo, capacidad integradora y vínculos de identidad con el entorno, y fomentando la creatividad, la experimentación y un ocio saludable)". Comarkalada se plantea a futuro "potenciar el proyecto introduciendo cambios para adaptarse a los cambios en los destinatarios, así como comprender más ámbitos de actuación como asociaciones, grupos de música, deportistas, etc., todo ello sin perder la cultura juvenil marca del proyecto".

\section{Conclusión}

Es necesario destacar cómo todos los proyectos presentados y analizados en este texto tienen una perspectiva de futuro muy consolidada y que pese a posible ajustes presupuestarios en las diferentes áreas, no prevén un descenso de su actividad, sino que debido en gran medida al éxito de participación de las personas jóvenes en sus programas, la dinámica creada les permite afrontar nuevos retos.

Esta alta tasa de participación juvenil en estos programas se debe en gran medida a que, tal y como señalan Ortega et al. (2015), sean los propios jóvenes los que establecen las normas y se autogestionen. La motivación que esto supone, permite que la experiencia del joven sea más auténtica y un margen a la experimentación tan necesaria en esta etapa. Las personas jóvenes viven estas experiencias como propias y el papel que juega la administración, más que como de ofertante que vende un producto, se percibe como un acompañante, un mediador o incluso un confidente. Este vínculo favorece la generación de múltiples dinámicas de participación y construcción social, política y cultural del joven. Pero de partida, el compromiso no sólo debe ser del joven, sino que la institución que promueve estas acciones (generalmente administración local) también debe demostrar el compromiso con el proyecto que se plantea de forma clara y transparente.

Por lo tanto, se puede señalar que una mayor promoción por parte de la juventud de sus experiencias de ocio, así como una mayor participación en la gestión, diseño, ejecución y evaluación de los espacios y servicios supone una mayor implicación. De lo contrario, lo que se puede encontrar son actividades de carácter más puntual para la reunión de personas jóvenes y que pudiera tener como principal objetivo el conocer gente nueva. Así lo afirman las instituciones de las cuales se han tomado las buenas prácticas, ya que muchas de ellas no entienden su proyecto sin la necesaria participación del joven a todos los niveles.

Como línea de futuro, se plantea la posibilidad de realizar una lectura en profundidad desde la teoría de ocio valioso (Cuenca, 2014), desde las experiencias vinculadas a los planteamientos de intervención/participación juvenil, los espacios donde se interviene y la propuesta de oferta de ocio desarrollada.

En ese sentido, la búsqueda de nuevas experiencias para poder ampliar el trabajo de campo y consolidar aspectos que se han destacado en el apartado de análisis, sería realmente interesante. Además, resultaba de gran utilidad realizar una ampliación del 
trabajo de investigación cooperativo tanto entre diferentes universidades como de los agentes que intervienen de una u otra forma en la juventud, Ilegando más allá de la elaboración de unas fichas con una serie de criterios: realizando encuentros y técnicas de trabajo en grupo. De esta manera se afinarían mucho más los aspectos destacados en el análisis presentado.

Uno de los ejes de futuro en este sentido, podría hacer referencia al análisis o estudio de los beneficios ligados a la autonomía y autogestión de los espacios juveniles de ocio, así como de la oferta de prácticas de ocio.

Podría ser interesante incluir el territorio donde estas acciones se asientan como una nueva variable que no ha sido tenida en cuenta, ya que la mayoría de las buenas prácticas analizadas hacen referencia a espacios urbanos. Conocer si un entorno más cercano como el que puede darse en un ámbito rural influye en los modos de organización y gobernanza del ocio podría ser otra línea de estudio interesante.

\section{REFERENCIAS BIBLIOGRÁFICAS}

Ahedo, R., Valdemoros, M.A., Escolar, M., Melendro, M., Serrat, N., y Pose, H. (2014). El valor de las buenas prácticas en la investigación en red. En G. Pérez y A. DeJuanas (Eds.) (2014), Educación y jóvenes en tiempos de cambio (pp. 219-226). Madrid: UNED.

Aristóteles (1970). Ética a Nicómaco. Traducido por M. Araujo y J. Marías. Madrid: Instituto de Estudios Políticos.

Berrio-Otxoa, K., Hernández, J.M., y Martínez, Z. (2002). Los adolescentes y el Tiempo Libre: Mirando al futuro. 2001-2002. Universidad del País Vasco.

Caride, J.A. (2012). Lo que el tiempo educa: el ocio como construcción pedagógica y social. ARBOR. Ciencia, Pensamiento y Cultura, 188, 301-313.

Caride, J.A., Fraguela, R., y Varela, A. (2014). Investigación en Red: Ios tiempos educativos y sociales, en clave de ocio y desarrollo humano. Investigación. Cultura, Ciencia y Tecnología (Instituto de Cultura, Ciencia y Tecnología, 6(11), 14-22.

Cuenca, M. (2000). Ocio humanista. Documentos de Estudios de Ocio, 16. Bilbao: Universidad de Deusto.

Cuenca, M. (2014). Ocio valioso. Documentos de Estudios de Ocio, 52. Bilbao: Universidad de Deusto.

Doistua, J.; Cuenca, M., y Ortega, C. (2014). Prácticas y experiencia de ocio en los jóvenes de la Comunidad Autónoma de Euskadi. En G. Pérez y A. De-Juanas (Eds.) (2014), Educación y jóvenes en tiempos de cambio (pp. 375-386). Madrid: UNED.

Gil Calvo, E. (2005). El envejecimiento de la juventud. Revista Estudios de Juventud, 71, 9-11.

González-Monteagudo, J. (2000). El paradigma interpretativo en la investigación social y educativa: nuevas respuestas para viejos interrogantes. Cuestiones pedagógicas: Revista de ciencias de la educación, 15, 227-246.

Tejerina, B., Carbajo, D., y Martínez, M. (2012). El fenómeno de las lonjas juveniles. Nuevos espacios de ocio y sociabilidad en Vitoria-Gasteiz. Informes del CEIC, 004. 
López, J.A. (2010). Ocio, consumo y medios de comunicación. En J. González-Anleo y P. González (Dir./Coord.) (2010). Jóvenes españoles 2010 (pp. 229-298). Madrid: Fundación SM.

Ortega, C., Lazcano, I., y Manuel, M. (2015). Espacios de ocio para jóvenes, de la monitorización a la autogestión. Pedagogía Social. Revista Interuniversitaria, 25, 69-8.

Rodríguez, E., Megías, I., y Sánchez, E. (2002). Jóvenes y relaciones grupales. Madrid: Obra Social de Caja Madrid. 